\title{
Is Prophylactic Central Compartment Neck Dissection Indicated for Clinically Node-Negative Papillary Thyroid Cancer: The Answer is Dependent on How the Data are Interpreted and the Weight Given to the Risks and Benefits
}

\author{
Christopher R. McHenry, MD \\ Department of Surgery, MetroHealth Medical Center, School of Medicine, Case Western Reserve University, Cleveland, \\ $\mathrm{OH}$
}

The article entitled "Prophylactic Central Compartment Neck Dissection in Papillary Thyroid Cancer and Effect on Locoregional Recurrence" by Hughes and colleagues is well-written and informative. ${ }^{1}$ The authors summarize the best data that currently exists in the literature regarding oncologic outcomes and complication rates for prophylactic central compartment neck dissection (CCND) in patients with clinically node-negative papillary thyroid cancer. The real value of this article is the analysis of the collective data from multiple studies. What remains controversial is how to interpret these data.

Interpretation of the data is dependent on the weight given to the risks and benefits of prophylactic CCND. The authors have documented a more than twofold higher risk of permanent hypoparathyroidism associated with total thyroidectomy and CCND compared with total thyroidectomy alone for clinically node-negative papillary thyroid cancer without any difference in locoregional recurrence. ${ }^{1}$ Based on the authors' interpretation of the data, they suggest that the benefits of prophylactic CCND for clinically node-negative papillary thyroid cancer is primarily to detect occult lymph node metastases, which may change the postoperative management with regard to radioactive iodine administration.

(C) Society of Surgical Oncology 2018

First Received: 24 June 2018;

Published Online: 16 July 2018

C. R. McHenry, MD

e-mail: cmchenry@metrohealth.org
The authors point out that in some studies, the demonstration of microscopic lymph node metastases resulted in higher rates of radioactive iodine administration and higher doses of radioactive iodine. ${ }^{1}$ However, high-level evidence to support the more aggressive use of radioactive iodine based on the results of prophylactic CCND is lacking. The efficacy of radioiodine for the treatment of lymph node micrometastases also remains unclear. What the authors do not consider is that prophylactic CCND may lead to the unnecessary administration of radioiodine in higher doses without apparent benefit and with the potential for radioiodine-induced morbidity.

In a review of the literature, Sawka and colleagues could not demonstrate a consistent significant benefit for iodine131 ablation in reducing recurrence or cause-specific mortality in early-stage papillary thyroid cancer. ${ }^{2}$ Furthermore, Hay and colleagues did not observe a significant reduction in recurrence at 20-year follow-up in patients with papillary thyroid cancer who had a MACIS score of $<6$ who were treated with radioiodine remnant ablation, regardless of their lymph node status at presentation. ${ }^{3,4}$ In a prospective study using propensity analysis for the evaluation of outcomes, published by the National Thyroid Cancer Treatment Cooperative Study Group, no significant benefit in overall survival, disease-specific survival, and disease-free survival was observed in patients with stage I papillary thyroid cancer who underwent radioiodine remnant ablation, although their mean follow-up was only 3 years. ${ }^{5}$ Nixon and colleagues from Memorial SloanKettering reported that following total thyroidectomy, the majority of patients with low-risk disease, and certain patients with advanced-stage disease (T3 tumors) or regional metastases, have low rates of recurrence and high 
survival rates without radioiodine ablation. ${ }^{6}$ It is important to emphasize that at least $80 \%$ of patients with papillary thyroid cancer have low-risk disease. It is also important to recognize that approximately $25 \%$ of papillary thyroid cancers do not concentrate radioiodine and the lack of iodine uptake increases with age. ${ }^{7}$ Furthermore, radioiodine is associated with potential morbidity, including salivary gland dysfunction, which can cause chronic dry mouth and throat, increased dental caries and loss of teeth, and recurrent sialadenitis. It can also cause ageusia (loss of taste), lacrimal gland dysfunction and nasolacrimal outflow obstruction, leukopenia, decreased sperm counts in men and ovarian dysfunction and menstrual irregularities in women, dysphagia, and second malignancies. ${ }^{8,9}$ It is also associated with increased financial cost. Currently, the only established indications for postoperative radioiodine for papillary thyroid cancer are the presence of systemic metastases, extrathyroidal extension, and tumors $>4 \mathrm{~cm} .{ }^{10}$

In summary, based on the collective data that Dr. Hughes and colleagues have summarized, prophylactic CCND does not reduce locoregional recurrence and is associated with a twofold increase in permanent hypoparathyroidism. It may have a role in helping select patients for radioiodine administration, but the current strength of the evidence to support this is expert opinion only and there are many experts who have shown no such benefit. It is important to recognize that there are inadequate and conflicting data to make definitive recommendations regarding radioiodine administration. Based on my interpretation of the data, the risks of prophylactic CCND outweigh the potential benefits. In my opinion, CCND should be limited to patients with macroscopic lymph node metastases.

\section{REFERENCES}

1. Hughes DT, Rosen JE, Evans DB, et al. Prophylactic central compartment neck dissection in papillary thyroid cancer and effect on locoregional recurrence. Ann Surg Oncol. 2018. http s://doi.org/10.1245/s10434-018-6528-0.

2. Sawka AM, Brierley JD, Tsang RW, Thabane L, Rotstein L, Gafni A, et al. An updated systematic review and commentary examining the effectiveness of radioactive iodine remnant ablation in well-differentiated thyroid cancer. Endocrinol Metab Clin N Am. 2008;37(2):457-80.

3. Hay ID, McConahey WM, Goellner JR. Managing patients with papillary thyroid carcinoma: insights gained from the Mayo Clinic's experience of treating 2,512 consecutive patients during 1940 through 2000. Trans Am Clin Climatol Assoc. 2002;113:241-60.

4. Hay ID. Selective use of radioactive iodine in the postoperative management of patients with papillary and follicular thyroid carcinoma. J Surg Oncol. 2006;94:692-700.

5. Jonklaas J, Sarlis NJ, Litofsky D, et al. Outcomes of patients with differentiated thyroid carcinoma following initial therapy. Thyroid. 2006;16:1229-42.

6. Nixon IJ, Ganly I, Patel SG, et al. The results of selective use of radioactive iodine on survival and on recurrence in the management of papillary thyroid cancer, based on Memorial SloanKettering Cancer Center risk group stratification. Thyroid. 2013; 23(6):683-94.

7. Durante C, Haddy N, Baudin E, Leboulleux S, Hartl D, Travagli JP, et al. Long-term outcome of 444 patients with distant metastases from papillary and follicular thyroid carcinoma: benefits and limits of radioiodine therapy. J Clin Endocrinol Metab. 2006;91:2892-899.

8. Sawka AM, Thabane L, Parlea L, Ibrahim-Zada I, Tsang RW, Brierley JD, et al. Second primary malignancy risk after radioactive iodine treatment for thyroid cancer: a systematic review and meta-analysis. Thyroid. 2009:19:451-57.

9. Almeida JP, Sanabria AE, Lima EN, Kowalski LP. Late side effects of radioactive iodine on salivary gland function in patients with thyroid cancer. Head Neck. 2011:33:686-90.

10. Haugen BR, Alexander EK, Bible KC, et al. 2015 Management guidelines for adult patients with thyroid nodules and differentiated thyroid cancer: the American Thyroid Association Guidelines Task Force on thyroid nodules and differentiated thyroid cancer. Thyroid. 2016;26(1):1-133. 